\title{
A Finite Dimensional Sliding Mode Observer for a Spatially Continuous Process
}

\author{
Mehmet Önder Efe
}

\begin{abstract}
Modeling and control of systems represented by Partial Differential Equations (PDEs) is an interesting research field as the process under investigation is infinite dimensional and most commonly used techniques are for finite dimensional systems. This paper considers the development of a finite dimensional observer obtained after an appropriate model reduction stage. A sliding mode observer is considered as it utilizes the sign of a quantity and ensures good reconstruction performance. The paper compares the fictitious state variables that are obtained after projecting the instantaneous snapshots on an eigenbasis and the state variables predicted by the observer. The results emphasize that the designed observer functions well on some set of operating conditions, which are elaborated in the paper.
\end{abstract}

\section{INTRODUCTION}

Processes modeled by PDEs have been in the focus of many researchers as they display quite complicated and rich sets of responses under different initial and boundary conditions. One such problem is to develop an observer extracting a finite number of dominant modes from a temporal evolution of a spatially continuous process. The essence of such an application is to alleviate the adverse effects of noise on the observed process output while providing a set of descriptive and useful state information.

The design of observers for finite dimensional linear systems is a mature field and well-developed schemes are available such as Kalman estimators. Lyapunov techniques are useful when the process under investigation is nonlinear, and further, the Lyapunov techniques can also be extended to the applications where the dynamics are governed by PDEs, as we consider here. In the first step, the process model undergoes an order reduction scheme to represent the essential dynamics with few Ordinary Differential Equations (ODEs). A widely used technique for the modal decomposition is Proper Orthogonal Decomposition (POD) with method of snapshots. This approach is first proposed by Lumley [1] to extract the dominant modes from turbulent flows, and Sirovich [2] improved it by introducing the method of snapshots and this technique has found many applications in flow modeling and control science, where the observed quantities are quite noisy in practice, and the use of an observer is oftentimes inevitable.

A low dimensional (LD) model, if it is achievable, may be useful for designing a LD observer. In this paper, a sliding mode observer (SMO) is considered to obtain the finite dimensional state information of a 2D heat flow characterized

Dr. Efe is with TOBB Economics and Technology University, Department of Electrical and Electronics Engineering, Sögütözü, Ankara, Turkey, Phone: +90-312-292-4064, Fax: +90-312-292 4180 onderefedieee. org by a parabolic PDE. The concept of SMO originates from the context of variable structure systems (VSS) theory in general and sliding mode control (SMC) in particular, [3], [4]. As a control technique, SMC is well known for its robustness against disturbances, [5]. The behavior emerges as a result of forcing the error vector towards a particular subspace of the state space, which is a stable locus having a unique equilibrium at the origin of the phase space. Despite the power of the method, chattering is a major problem of SMC systems since the sign of a quantity which is very close to zero is utilized. Nonetheless, the methods like sign function smoothing introduces a boundary layer with finite thickness offering a practical solution to the problem of chattering, [6]. Once designed, the LD model described above can be utilized as an observer quickly providing the state of the process. The crux of the design is to elaborate the conditions over which the observer functions appropriately.

This paper is organized as follows. In the second section, we present the POD, and POD based LD modeling strategy. Following this, we focus on the design and analysis of the LD SMO. The fourth section presents the simulation studies with particular emphasis on the operating conditions affecting the performance of the SMO. The concluding remarks are given at the end of the paper.

\section{ORder REDUCtion Via POD MEthod}

Let $U_{i}(x, y), i=1,2, \ldots, N_{s}$ be a set of entities, where $N_{s}$ is the number of elements. Every element of this set corresponds to a snapshot (instantaneous solution) observed from a process, say for example the $2 \mathrm{D}$ heat flow with initial and boundary conditions,

$$
\begin{aligned}
u_{t}(x, y, t) & =c^{2}\left(u_{x x}(x, y, t)+u_{y y}(x, y, t)\right) \\
u(x, 0, t) & =f(x) \gamma(t) \\
u(1, y, t) & =0, \quad u(x, 1, t)=0, \quad u(0, y, t)=0 \\
u(x, y, 0) & =0 \quad \forall(x, y)
\end{aligned}
$$

where, $c$ is the constant thermal diffusivity parameter that is known, $f(x)$ is the gain effective along the $y=0$ boundary segment, and the subscripts $x, y$ and $t$ refer to the partial differentiation with respect to $x, y$ and time, respectively. The continuous time process takes place over the physical domain $\Omega:=\{(x, y) \mid(x, y) \in[0,1] \times[0,1]\}$ and the solution is obtained on a spatial grid denoted by $\Omega_{d}$, which describes the coordinates of the pixels of every snapshot in the ensemble. The entities described over $\Omega_{d}$ are matrices in $\mathbb{R}^{N_{y} \times N_{x}}$. The 
goal is to find an orthonormal basis set letting us to write the solution as

$$
u(x, y, t)=\sum_{i=1}^{R_{L}} \alpha_{i}(t) \Phi_{i}(x, y)
$$

where $\alpha_{i}(t)$ is the $i^{\text {th }}$ temporal mode, $\Phi_{i}(x, y)$ is the $i^{\text {th }}$ spatial function (basis functions or the eigenbasis) defined over $\Omega, R_{L}$ is the number of independent basis functions that can be synthesized from the given ensemble, or equivalently that spans the space described by the ensemble. If the basis set $\left\{\Phi_{i}(x, y)\right\}_{i=1}^{R_{L}}$ is an orthonormal set, Galerkin projection yields the autonomous Ordinary Differential Equations (ODEs) directly. The POD procedure utilized in this study is described in [7] in detail.

Fundamental Assumption: The majority of works dealing with POD and model reduction applications presume that the flow (the solution of the PDE process) is dominated by coherent modes. Because of the dominance of coherent modes, the typical spread of the eigenvalues of the correlation matrix turns out to be logarithmic and the terms decay very rapidly in magnitude. This fact enables us to assume that a reduced order representation, say with $M<R_{L}$ modes can also be written as an equality

$$
u(x, y, t)=\sum_{i=1}^{M} \alpha_{i}(t) \Phi_{i}(x, y)
$$

and the reduced order model is derived under the assumption that (3) satisfies the governing PDE in (1), (See [7], [8], [9]). Unsurprisingly, such an assumption results in a model having uncertainties, however, one should keep in mind that the goal is to find a model, which matches the infinite dimensional system in some sense of approximation with typically $M \ll$ $R_{L} \leq N_{s}$, where $R_{L}$ denotes the rank of the correlation matrix $L$. To represent how good such an expansion is, a percent energy measure is defined as follows

$$
E=\frac{\sum_{i=1}^{M} \lambda_{i}}{\sum_{i=1}^{R_{L}} \lambda_{i}} \times 100 \%
$$

where the tendency of $E \rightarrow 100 \%$ means that the model captures the dynamical information contained in the snapshots well. Conversely, an insufficient model will be obtained if $E$ is far below $100 \%$. In above, $\lambda_{i}$ stands for an eigenvalue of $L$.

The second part of the fundamental assumption emphasizes the discretization of the PDE. Since the numerical solver computes the behavior of the process over a grid having finite number of pixels, we assume that the solution obtained over the computational grid is descriptive enough to admit the solution as a snapshot from the process.

In the order reduction phase, we need to obtain the autonomous ODE model first. Towards this goal, if (3) is a solution to the PDE in (1), then it has to satisfy the PDE. Substituting (3) into (1) with the fundamental assumption yields

$$
\sum_{i=1}^{M} \dot{\alpha}_{i}(t) \Phi_{i}(x, y)=c^{2} \sum_{i=1}^{M} \alpha_{i}(t) \Psi_{i}(x, y)
$$

where $\Psi_{i}(x, y)=\frac{\partial^{2} \Phi_{i}(x, y)}{\partial x^{2}}+\frac{\partial^{2} \Phi_{i}(x, y)}{\partial y^{2}}$. Taking the inner product of both sides with $\Phi_{k}(x, y)$ and remembering $\left\langle\Phi_{i}(x, y), \Phi_{k}(x, y)\right\rangle_{\Omega}=\delta_{i k}$ with $\delta_{i k}$ being Kronecker delta yields

$$
\dot{\alpha}_{k}(t)=c^{2} \sum_{i=1}^{M} \alpha_{i}(t)\left\langle\Phi_{k}(x, y), \Psi_{i}(x, y)\right\rangle_{\Omega}
$$

Defining $\zeta_{k}$ as the entity in $\Omega_{d}$ corresponding to the entity $\Psi_{k}$ in $\Omega$, one could rewrite (6) as

$$
\dot{\alpha}_{k}(t)=c^{2} \sum_{i=1}^{M} \alpha_{i}(t)\left\langle\phi_{k}, \zeta_{i}\right\rangle_{\Omega_{d}}
$$

Let $\mathcal{A}, \mathcal{B} \in \mathbb{R}^{N_{y} \times N_{x}}$. Define the inner product seen in (7) as $\mathcal{A} \star \mathcal{B}:=\frac{1}{N_{s}} \sum_{i=1}^{N_{y}} \sum_{j=1}^{N_{x}} \mathcal{A}(i, j) \mathcal{B}(i, j)$ The equation in (7) can be written explicitly by using $\star$ operator as

$$
\dot{\alpha}_{k}(t)=c^{2} \sum_{i=1}^{M} \alpha_{i}(t)\left(\phi_{k}(x, y) \star \zeta_{i}(x, y)\right)
$$

Notice that $\star$ operator can be applied over nonoverlapping subdomains of $\Omega_{d}$. This lets us separate the entries corresponding to boundaries without modifying the values of $\phi_{k}(x, y) \star \zeta_{i}(x, y)$ as seen in (9),

$$
\begin{array}{r}
\dot{\alpha}_{k}(t)=\quad c^{2} \sum_{i=1}^{M} \alpha_{i}(t)\left(\phi_{k}(x, 0) \star \zeta_{i}(x, 0)\right)+ \\
c^{2} \sum_{i=1}^{M} \alpha_{i}(t)\left(\phi_{k}^{\circ}(x, y) \star \zeta_{i}^{\circ}(x, y)\right)
\end{array}
$$

In above, $\phi_{k}^{\circ}(x, y)$ denotes a matrix that is obtained when the $y=0$ boundary elements of $\phi_{k}(x, y)$ are removed. The $k^{\text {th }}$ component of the first summation above, which is obtained when $i=k$, can be separated from the expression and we obtain (10), which let us embed the boundary conditions into the expression,

$$
\begin{aligned}
\dot{\alpha}_{k}(t)= & c^{2} \alpha_{k}(t) \phi_{k}(x, 0) \star \zeta_{k}(x, 0)+ \\
& c^{2} \sum_{i=1}^{M} \alpha_{i}(t)\left(1-\delta_{i k}\right) \phi_{k}(x, 0) \star \zeta_{i}(x, 0)+ \\
& c^{2} \sum_{i=1}^{M} \alpha_{i}(t)\left(\phi_{k}^{\circ}(x, y) \star \zeta_{i}^{\circ}(x, y)\right)
\end{aligned}
$$

At this stage of the modeling, we need to restate the boundary conditions in such a way that the final expression above can be incorporated with these conditions. The underlying idea is straightforward: If (3) is a solution to the PDE in (1), then is must be satisfied at the boundaries as well. This lets us write the following 


$$
\sum_{i=1}^{M} \alpha_{i}(t) \phi_{i}(x, 0)=f(x) \gamma(t)
$$

which can be rewritten as

$$
\alpha_{k}(t) \phi_{k}(x, 0)=f(x) \gamma(t)-\sum_{i=1}^{M}\left(1-\delta_{i k}\right) \alpha_{i}(t) \phi_{i}(x, 0)
$$

The expression above can be inserted into the first line of (10) and we explicitly see $\gamma(t)$, and the following expression is obtained.

$$
\begin{aligned}
\dot{\alpha}_{k}(t)= & c^{2}\left(f(x) \star \zeta_{k}(x, 0)\right) \gamma(t)+ \\
& c^{2} \sum_{i=1}^{M} \alpha_{i}(t)\left(\phi_{k} \star \zeta_{i}-\phi_{i}(x, 0) \star \zeta_{k}(x, 0)\right)
\end{aligned}
$$

which can be written compactly as

$$
\dot{\alpha}(t)=A \alpha(t)+B \gamma(t)
$$

where $\alpha(t)=\left(\begin{array}{llll}\alpha_{1}(t) & \alpha_{2}(t) & \ldots & \left.\alpha_{M}(t)\right)^{\mathrm{T}} \text { and }\end{array}\right.$

$$
A_{k i}=c^{2}\left(\phi_{k}(x, y) \star \zeta_{i}(x, y)-\phi_{i}(x, 0) \star \zeta_{k}(x, 0)\right)
$$

and the $k^{\text {th }}$ row of the input vector is

$$
B_{k}=c^{2}\left(f(x) \star \zeta_{k}(x, 0)\right)
$$

This result practically lets us have a non-autonomous linear dynamical model for the infinite dimensional process in (1), which constitutes a basis for the finite dimensional SMO.

A natural question is whether this model recovers the unforced dynamics, which is obtained by setting $\gamma(t)=0$, and the steady state dynamics obtained when $\dot{\alpha}(t)=0$. Straightforward manipulations will show that the dynamics in (6) is recovered in both cases.

As seen clearly, once the finite dimensional model for an infinite dimensional process is obtained, one might implement an observer to obtain a useful state information, which is discussed in the sequel.

\section{SLiding Mode ObServer}

First we demonstrate that a sliding mode PDE observer can be proposed while maintaining the stability. Consider the following process

$$
\begin{aligned}
v_{t}(x, y, t) & =c^{2}\left(v_{x x}(x, y, t)+v_{y y}(x, y, t)\right)+\mathcal{K} \operatorname{sgn}(u-v) \\
v(x, 0, t) & =f(x) \gamma(t) \\
v(1, y, t) & =0, v(x, 1, t)=0, \quad v(0, y, t)=0
\end{aligned}
$$

where $\mathcal{K}>0$ stands for the observer gain and we choose the following Lyapunov function candidate to study the stability

$$
V=\frac{1}{2} \int_{0}^{1} \int_{0}^{1}(u-v)^{2} \mathrm{~d} x \mathrm{~d} y
$$

and evaluate its time derivative as seen below,

$$
\begin{aligned}
\dot{V}= & \int_{0}^{1} \int_{0}^{1}(u-v)\left(u_{t}-v_{t}\right) \mathrm{d} x \mathrm{~d} y \\
= & -\mathcal{K} \int_{0}^{1} \int_{0}^{1}|z| \mathrm{d} x \mathrm{~d} y+ \\
& c^{2} \int_{0}^{1} \int_{0}^{1} z\left(z_{x x}+z_{y y}\right) \mathrm{d} x \mathrm{~d} y
\end{aligned}
$$

where $z:=u-v$ is the switching variable. Since the process under investigation is first order, $z=0$ is the sliding manifold for each $(x, y) \in \Omega$. It is straightforward to show that

$$
\begin{aligned}
\int_{0}^{1} \int_{0}^{1} z z_{x x} \mathrm{~d} x \mathrm{~d} y & =\int_{0}^{1}\left(\left.z z_{x}\right|_{x=0} ^{x=1}-\int_{0}^{1} z_{x}^{2} d x\right) \mathrm{d} y \\
& =-\int_{0}^{1} \int_{0}^{1} z_{x}^{2} \mathrm{~d} x \mathrm{~d} y
\end{aligned}
$$

which is due to the fact that $z(1, y, t) z_{x}(1, y, t)-$ $z(0, y, t) z_{x}(0, y, t)=0$ as $z(1, y, t)=u(1, y, t)-$ $v(1, y, t)=0$ and $z(0, y, t)=u(0, y, t)-v(0, y, t)=0$ Likewise we have

$$
\int_{0}^{1} \int_{0}^{1} z z_{y y} \mathrm{~d} x \mathrm{~d} y=-\int_{0}^{1} \int_{0}^{1} z_{y}^{2} \mathrm{~d} x \mathrm{~d} y
$$

which is due to the fact that $z(x, 1, t) z_{x}(x, 1, t)$ $z(x, 0, t) z_{x}(x, 0, t)=0$ as $z(x, 1, t)=u(x, 1, t)-$ $v(x, 1, t)=f(x) \gamma(t)-f(x) \gamma(t)=0$ and $z(x, 0, t)=$ $u(x, 0, t)-v(x, 0, t)=f(x) \gamma(t)-f(x) \gamma(t)=0$.

Under these conditions, (17) becomes

$$
\dot{V}=-\mathcal{K} \int_{0}^{1} \int_{0}^{1}|z| \mathrm{d} x \mathrm{~d} y-c^{2} \int_{0}^{1} \int_{0}^{1}\left(z_{x}^{2}+z_{y}^{2}\right) \mathrm{d} x \mathrm{~d} y<0
$$

Since $\dot{V}<0$ is guaranteed, $v(x, y, t) \rightarrow u(x, y, t)$ as $t \rightarrow$ $\infty$, i.e. $v(x, y, t)$ reconstructs $u(x, y, t)$ in a globally stable fashion. If the process state $(u(x, y, t))$ is noisy, the observer filters out the noise and enables us to have a representative and finite dimensional state information.

An interesting question here is whether this observer is useful for feedback control purposes. Towards this goal, recall the fundamental assumption, stating that the $M$ term summation can be assumed as the true solution and the error is negligible. This lets us write $v(x, y, t)=$ $\sum_{i=1}^{M} \beta_{i}(t) \Phi_{i}(x, y)$, i.e. the dynamical constituents of the observer are projected onto the spatial eigenfunctions derived in the modeling stage, and this lets us have $M$ dimensional observer state, $\beta(t)$. Substituting above expression of $v(x, y, t)$ into the PDE in (15) yields

$$
\begin{aligned}
& \sum_{i=1}^{M} \dot{\beta}_{i}(t) \Phi_{i}(x, y)=c^{2} \sum_{i=1}^{M} \beta_{i}(t) \Psi_{i}(x, y)+ \\
& \mathcal{K} \operatorname{sgn}\left(u(x, y, t)-\sum_{i=1}^{M} \beta_{i}(t) \Phi_{i}(x, y)\right)
\end{aligned}
$$


where $\beta_{i}(t)$ is the temporal part corresponding to spatial eigenbasis $\Phi_{i}(x, y)$. Taking the inner product of both sides with $\Phi_{k}(x, y)$ lets us obtain (22),

$$
\dot{\beta}_{k}(t)=c^{2} \sum_{i=1}^{M} \beta_{i}(t)\left\langle\Phi_{k}(x, y), \Psi_{i}(x, y)\right\rangle_{\Omega}+\mathcal{K} \sigma(t)
$$

where $\sigma(t)=\left(\begin{array}{llll}\sigma_{1}(t) & \sigma_{2}(t) & \ldots & \left.\sigma_{M}(t)\right)^{\mathrm{T}} \text { and }\end{array}\right.$

$$
\begin{aligned}
\sigma_{k}(t) & =\left\langle\operatorname{sgn}\left(u-\sum_{i=1}^{M} \beta_{i}(t) \Phi_{i}(x, y)\right), \Phi_{k}(x, y)\right\rangle_{\Omega} \\
& =\left\langle\operatorname{sgn}\left(U_{t}-\sum_{i=1}^{M} \beta_{i}(t) \phi_{i}\right), \phi_{k}\right\rangle_{\Omega_{d}}
\end{aligned}
$$

where $U_{t} \in \Omega_{d}$ is the snapshot of the process at time $t$. Following the modeling procedure discussed before, we end up with the LD observer given below

$$
\dot{\beta}(t)=A \beta(t)+B \gamma(t)+\mathcal{K} \sigma(t)
$$

where $A$ and $B$ have been defined in (13)-(14). One should note that the computation of the term in (23) entails an infinite dimensional output feedback from the process, $U_{t}$, which is projected onto the eigenbasis, $\phi_{k}$, thereby forming a finite dimensional state information that is the counterpart of $\alpha_{k}(t):=\left\langle U_{t}, \phi_{k}\right\rangle_{\Omega_{d}}$.

A last remark in this section is about meaning of the Lyapunov function in (16). Under the presence of the fundamental assumption, one can write the equalities in (25), which tells us that the infinite dimensional form of the Lyapunov function is mapped to a well-known Lyapunov function in $\mathbb{R}^{M}$. Therefore the proof of negative definiteness of one would clearly imply the other as long as the fundamental assumption holds true. In the next section, we consider the boundary control of the system by using the state information from the LD observer.

$$
\begin{aligned}
V_{o} & =\frac{1}{2} \int_{0}^{1} \int_{0}^{1}(u-v)^{2} \mathrm{~d} x \mathrm{~d} y \\
& =\frac{1}{2} \int_{0}^{1} \int_{0}^{1}\left(\sum_{i=1}^{M} \alpha_{i} \Phi_{i}-\sum_{i=1}^{M} \beta_{i} \Phi_{i}\right)^{2} \mathrm{~d} x \mathrm{~d} y \\
& =\frac{1}{2} \int_{0}^{1} \int_{0}^{1}\left(\sum_{i=1}^{M} e_{i} \Phi_{i}\right)^{2} \mathrm{~d} x \mathrm{~d} y \\
& =\frac{1}{2} \int_{0}^{1} \int_{0}^{1}\left(\sum_{i=1}^{M} e_{i}^{2} \Phi_{i}^{2}+\sum_{i=1}^{M} \sum_{j=1, j \neq i}^{M} e_{i} e_{j} \Phi_{i} \Phi_{j}\right) \mathrm{d} x \mathrm{~d} y \\
& =\frac{1}{2} e^{\mathrm{T}} e^{(25)}
\end{aligned}
$$

\section{Simulation Results}

In the simulations, we have solved the PDE in (1) with Crank-Nicholson algorithm. The numerical grid has $N_{x}=$ $30, N_{y}=30$ points in $x$ and $y$ directions, final time is $T=0.5 \mathrm{sec}$. and the simulation step size in $50 \mu \mathrm{s}$. The PDE has $f(x)=\sin (2 \pi x)$ as the boundary gain effective along $y=0$ segment of $\Omega$ and $c=1$ as the thermal diffusivity parameter. The boundary excitation signal utilized during the model derivation is given by

$$
\gamma(t)=\sin (2 \pi 50 t(T-t))
$$

which is a spectrally rich signal exciting the modes of the infinite dimensional process. The first four modes $(M=4)$ have been chosen and linearly sampled 251 snapshots out of 1001 were used in the derivation of the LD model. For these choices, $\mathrm{E}=99.9989 \%$ of the total energy contained in the samples is preserved in the LD model.

In simulating the process, we have embedded the PDE solver into the Matlab/Simulink ${ }^{\circledR}$ environment. We investigate the quantity given by

$$
Q(t):=\sqrt{\sum_{k=1}^{M}\left(\alpha_{k}(t)-\beta_{k}(t)\right)^{2}}
$$

The reason why we check this quantity is to figure out the observer's performance. The smaller the squared norm the closer the states of the observer to the equivalent process states.

The signals shown in the figure have been perturbed additively by zero mean Gaussian noise sequences having noise power 0.002 and the observer receives the noisy information from the process. This scenario is implemented to assess the performance of the observer under realistic operating conditions.
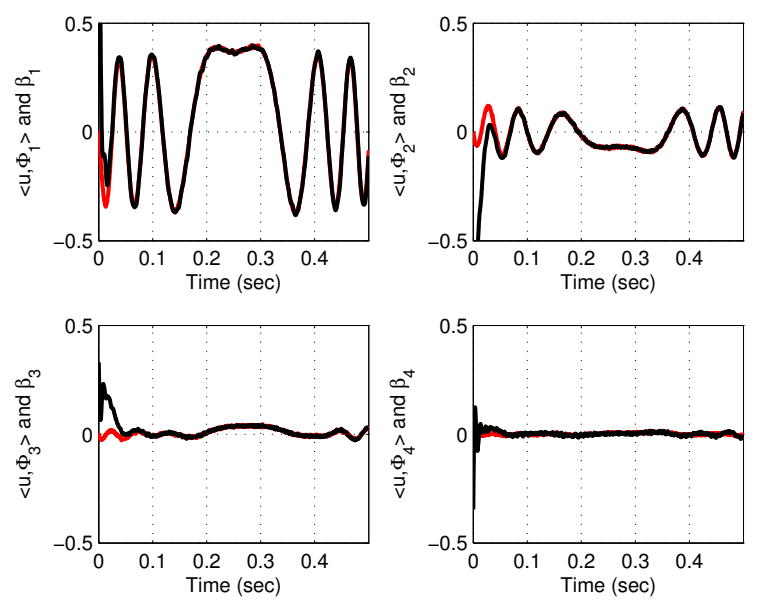

Fig. 1. The evolution of the variables in time domain

In Fig. 1, the response of the observer, which is the set of $M \times 1$-dimensional state vector, and the corresponding quantities computed by the inner product $\left\langle u(x, y, t), \Phi_{i}(x, y)\right\rangle_{\Omega}$, $i=1,2,3,4$ are illustrated. The observer gain $\mathcal{K}$ is selected as 1 and a very good reconstruction performance is observed. As the value of $\mathcal{K}$ is increased, quicker convergence can be observed, alternatively, smaller values lead to slower convergence speed. The value here was chosen by trial and 

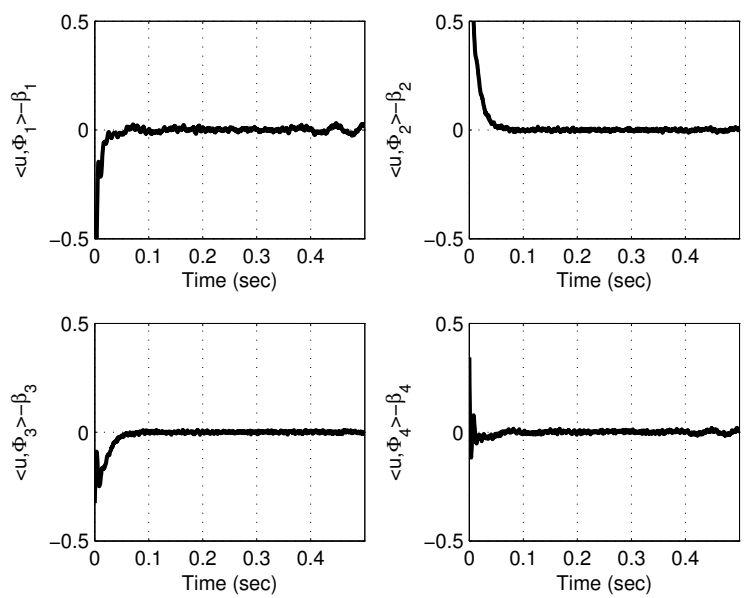

Fig. 2. The evolution of the observer errors

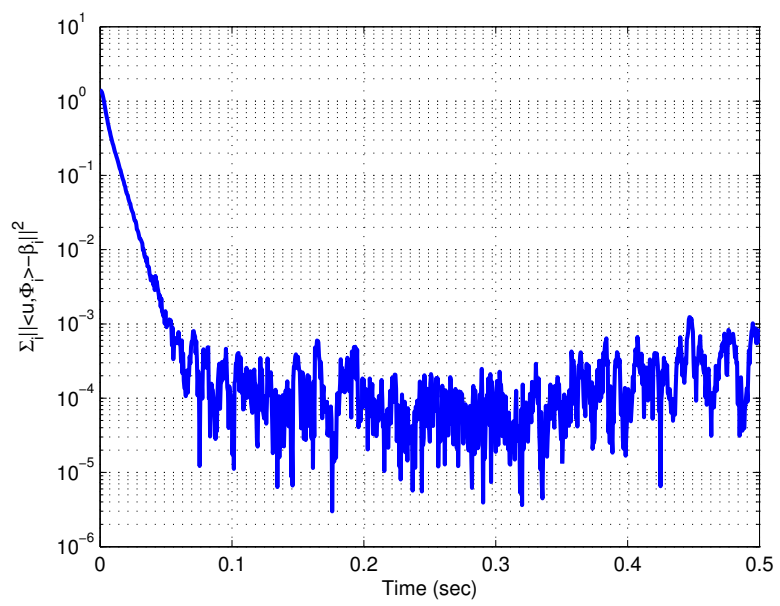

Fig. 3. Convergence in the initial transient and dependence of performance on the spectral content

error. As seen from Fig. 1, the initial state of the observer, $\beta(0)=(10,-7,3,-1)^{\mathrm{T}}$ is driven towards its desired value very quickly. The two curves in each subplot are almost indistinguishable and this is a good indicator of performance.

Fig. 2 illustrates the difference between the states constructed by the LD SMO and the desired values. The temporal evolution is close to zero in all four states. Another observation in this figure is the evolution of the error in terms of the spectral content of the boundary excitation. Clearly the external excitation given by (26) has relatively higher frequencies at times close to zero and $T$, and the errors seen in Fig. 2 have a tendency to increase when the boundary excitation has components at higher frequencies, which is particularly visible in the top left subplot of Fig. 2. This will be explored further in the below discussion.

In Fig. 3, the convergent nature of the initial behavior as well as the above emphasized issue of spectral dependence is seen compactly for all four error terms. The quantity plotted
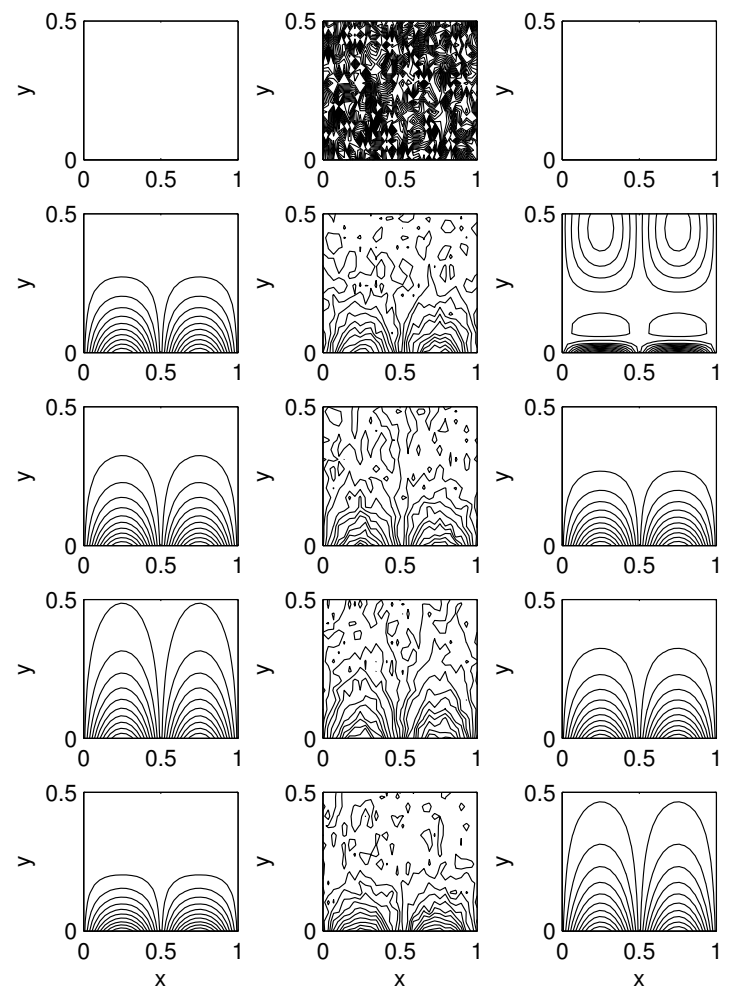

Fig. 4. The system output (left), noisy output as an input to the observer (middle) and the output reconstructed via states provided by the sliding mode observer

in this figure is defined in (27). Clearly the observer functions well when the excitation signal changes slowly.

Fig. 4 depicts some snapshots from the noiseless process output, noisy process output, which is an input to the observer, and the response of the observer with the available eigenbasis are shown at period of $100 \mathrm{msec}$. The results emphasize that the observer functions well in removing the spurious content caused by the noise components and provides a useful state information in finite dimensions.

The last issue in this section is the study of determining the performance of the observer when the spectral qualities of the input signal changes. We test the performance of the observer with $\gamma(t)=\sin \left(2 \pi f_{c} t\right)$ and consider $1 \mathrm{~Hz}, 2 \mathrm{~Hz}$, $5 \mathrm{~Hz}, 10 \mathrm{~Hz}, 20 \mathrm{~Hz}, 50 \mathrm{~Hz}$ and $100 \mathrm{~Hz}$ as $f_{c}$. Furthermore, we explore the performance with varying powers of noise sequences corrupting the process output and the observer input. For these experiments, we consider the noise powers $p_{n_{i}}=p_{n_{o}} \in 0.002,0.02,0.2,2$. In Fig. 5, a bar chart is given to summarize the obtained results. The height of each bar is the supremum value of the quantity in (27) measured after the end of the initial transient regime, approximately after the first $100 \mathrm{msec}$.

The results seen in the figure stipulate that for low noise powers and for excitation signals having small $f_{c}$, the presented SMO displays outstanding performance. As the 


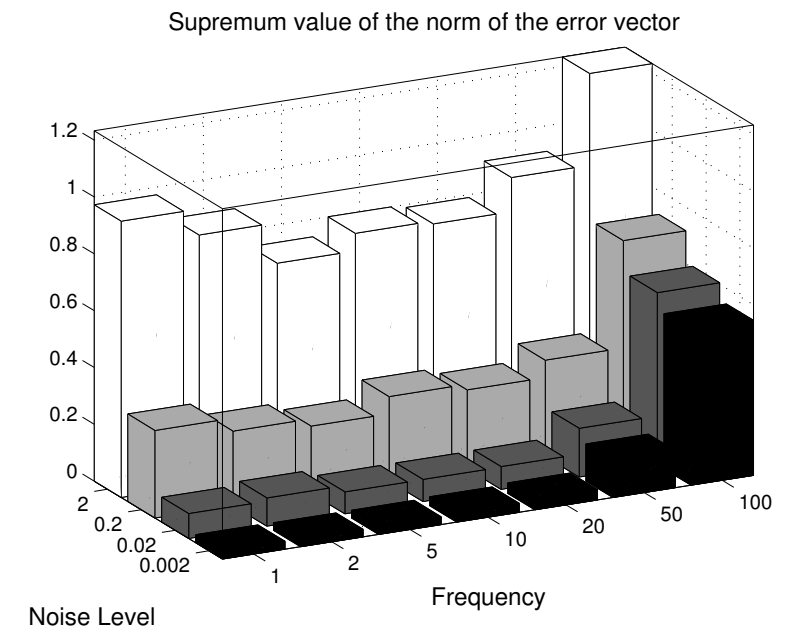

Fig. 5. The dependence of the observer performance on the noise level and the excitation frequency

frequency increases, the performance decreases gradually. Except those obtained with $p_{n_{i}}=p_{n_{o}}=2$, all results up to $f_{c}=50 \mathrm{~Hz}$ are quite promising. If one draws the Fast Fourier Transform (FFT) plot of the signal used during the model derivation (see (26)), it is seen that the signal has its dominant components mostly below $50 \mathrm{~Hz}$. This empirical result emphasizes the usefulness of the LD SMO for excitations that have dominant spectral content approximately below $50 \mathrm{~Hz}$.

\section{Conclusions}

This paper focuses on the design of a low dimensional (LD) sliding mode observer. First a LD model of the process under investigation is obtained and it is assumed that the LD model matches the process response precisely. The boundary term is separated in the autonomous LD model such that the LD model has an external input explicitly. This procedure yields a set of basis functions, called eigenbasis, and a finite dimensional ODE synthesizing the temporal content. An infinite dimensional (PDE) observer is proposed afterwards and utilizing the constructed eigenbasis, a LD counterpart of the SMO is obtained. Utilizing the Lyapunov stability conclusions, the paper demonstrates the stability and equivalence issues under the fundamental assumption.

The first important issue regarding the entire design effort is the role of the fundamental assumption, which is the underlying fact in most POD based modeling studies. This assumption emphasizes that the flow is dominated by coherent modes typically satisfied in the heat flow problems. The latter part is further crucial as it states that the results obtained from the numerical solver are admissibly descriptive. from a practical point of view, the assumption makes the LD model achievable yet with some uncertainties depending on the chosen number of modes $(M)$.

The second important conclusion is the dependence of the SMO's performance on the operating conditions. It is seen in the simulations that the observer performance degrades if the boundary excitation has high frequency components. This particularly stems form the fact that the LD observer is generated from the LD model of the process, which depends upon the selection of boundary excitations. If the excitations leading to the LD model are spectrally rich, then the obtained LD model performs well for the excitations that fall within the spectral coverage of the signal used during the derivation of the LD model.

Lastly, the choice of the number of modes $(M)$ deserves some attention. Increasing the number of modes in POD method may contribute to the performance of the LD SMO, however, as the mode number increases, the contribution of the newly added state is expected to be much smaller than the smallest of the available ones. This because of the typical logarithmic decay of the eigenvalues of the correlation matrix $L$. Besides, the addition of new states to the LD model or SMO will contribute to the computational complexity inevitably.

Overall, the paper introduces a LD SMO performing well with slowly changing excitations. The LD SMO has four states, which emphasizes the simplicity.

\section{Acknowledgments}

This work was supported in part Turkish Scientific Council (TÜBITAK) Contract 107E137 and by TOBB Economics and Technology University, BAP Program, under contract no ETÜ-BAP-2006/04.

The author gratefully acknowledges the facilities of TOBB ETÜ library and the constructive suggestions of the anonymous reviewers of the paper.

\section{REFERENCES}

[1] Lumley, J., "The structure of inhomogeneous turbulent flows," Atmospheric Turbulence and Wave Propagation, Nauca, Moscow, pp.166176, 1967.

[2] Sirovich, L., "Turbulence and the dynamics of coherent structures," Quarterly of Applied Mathematics, XLV, no. 3, pp. 561-590, 1987.

[3] Chen, W. and Saif, M., "Novel Sliding Mode Observers for a Class of Uncertain Systems," Proceedings of the 2006 American Control Conference, Minneapolis, Minnesota, USA, June 14-16, pp.26222627, 2006.

[4] Xie, W.-F., "Sliding-Mode-Observer-Based Adaptive Control for Servo Actuator With Friction," IEEE Transactions on Industrial Electronics, 54, n.3, pp.1517-1527, June 2007.

[5] Young, K.D., Utkin, V.I. and Özguner, Ü., "A Control Engineer's Guide to Sliding Mode Control," IEEE Trans. on Control Systems Technology, 7, n.3, pp. 328-342, May 1999.

[6] Slotine, J.-J. E. and Li, W., Applied Nonlinear Control, Prentice-Hall, New Jersey, 1991.

[7] Ly, H.V. and Tran, H.T., "Modeling and control of physical processes using proper orthogonal decomposition," Mathematical and Computer Modelling of Dynamical Systems, 33, pp.223-236, 2001.

[8] Rowley, C.W., Colonius, T., Murray, R.M., "Model reduction for compressible flows using POD and Galerkin projection," Physica DNonlinear Phenomena, 189 (1-2), pp.115-129, 2004.

[9] Rowley, C.W., "Model Reduction for Fluids, Using Balanced Proper Orthogonal Decomposition," International Journal of Bifurcation and Chaos, 15 (3), pp.997-1013, 2005. 